Distribution Category:

Mathematics and Computers (UC-32)

ANL $-81-7$

ARGONNE NATIONAL LABORATORY

9700 South Cass Avenue

Argonne, Illinois 60439

THE EFFECTS OF CURVATURE

ON ASYMMETRIC STEADY STATES

IN CATALYST PARTICLES

by

Bradley J. Lucier*

Applied Mathematics Division

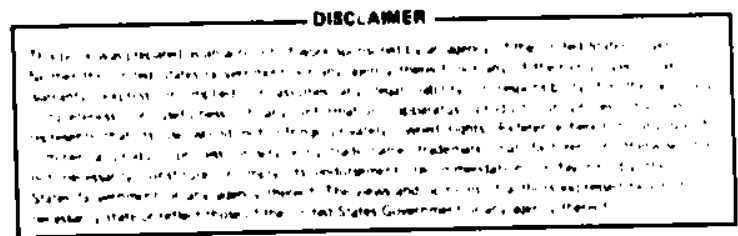

February 1981

`Current Address: Dept. of Mathematics, U. of Chicago, Chicago, IL 60637. 


\section{TABLE OF CONTENTS}

Abstract

The Model

Construction of Solutions

Numerical Results

Stability

Limitinq Cases

Acknowledqement

References

\section{LIST OF FIGURES}

1 Solutions of $(9),(10)$ with $K_{2}=50, k_{1}=12.594684 \ldots \ldots \ldots \ldots \ldots \ldots 17$

2 Solutions of (9),(10) with $K_{2}=50, k_{1}=12.598694 \ldots \ldots \ldots \ldots \ldots \ldots 18$

3 Solutions of $(9),(10)$ with $k_{2}=50, k_{1}=12.602673 \ldots \ldots \ldots \ldots \ldots \ldots 19$

4 Solutions of $(9),(10)$ with $k_{2}=50, k_{1}=12.765120 \ldots \ldots \ldots \ldots \ldots \ldots 20$

5 Solutions of $(9),(10)$ with $k_{2}=50, k_{1}=12.928872 \ldots \ldots \ldots \ldots \ldots \ldots \ldots 21$

6 Solutions of $(9),(10)$ with $k_{2}=5$ ?, $k_{1}=12.931540 \ldots \ldots \ldots \ldots \ldots \ldots 22$

7 Solutions of $(9),(10)$ with $K_{2}=50, K_{1}=12.934197 \ldots \ldots \ldots \ldots \ldots \ldots 23$

1 . Solutions of (9),(10) with $K_{2}=50, K_{1}=12.594684 \ldots \ldots \ldots \ldots \ldots \ldots 24$

2. Solutions of (9),(10) with $K_{2}=50, K_{1}=12.598694 \ldots \ldots \ldots \ldots \ldots \ldots 25$

3 . Solutions of $(9),(10)$ with $k_{2}=50, K_{1}=12.602673 \ldots \ldots \ldots \ldots \ldots \ldots . \ldots 26$

4 . Solutions of $(9),(10)$ with $K_{2}=50, K_{1}=12.765120 \ldots \ldots \ldots \ldots \ldots 27$ 


\title{
THE EFFECTS OF CURVATURE \\ ON ASYMMETRIC STEADY STATES \\ IN CATALYST PARTICLES
}

by

Bradley J. Lucier

\begin{abstract}
The effects of curvature on steady states of chemical catalytic reactions is investigated by studying the cases of the catalytic particle being a spherical or cylindrical shell. Existence and stability of solutions are studied. It is shown that the solutions converge to the solutions for the catalytic slab when the curvature goes to 0 in each case.
\end{abstract}

The Model

We will use a model for catalyst particles proposed elsewhere (see $[1],[3])$. We assume that the particle is a homogeneous porous body, and that a catalyst is on the surface of this bodv. We are interested in the concentration of a limiting substance in a chemical reaction which occurs on the surface of the particle because of the catalyst present there. The limitina substance can diffuse through the particle, and its concentration outside of the particle is kept constant and uniform. We assume the reaction rates do not vary with time.

If we denote the concentration of the limiting substance by $C$, the diffusion coefficient by $D$, the coefficient of mass transfer at the surface by $k$, and the concentrations of the limiting substance and the model reaction rates at the various surfaces of the particle (the inner and outer surfaces of a cylindrical shell, for example) by $C_{j}$ and $r_{j}(C)$, respectively, we find that $C$ satisfies the differential equation

$$
\frac{\partial C}{\partial t}{ }^{\star}=\text { DAC inside the particle } \Omega^{\star} \text {, }
$$

$$
\begin{aligned}
& C(x, y, z, 0)=C_{0}(x, y, z) \\
& \frac{D}{x} \frac{\partial C}{\partial n}+C-C_{j}+r_{j}(C)=0 \text { for each boundary surface } \partial \Omega_{j}^{\star} .
\end{aligned}
$$


Here $\frac{\partial C}{\partial n}$ denotes the derivative of $C$ in the direction normal to $\partial \Omega_{j}$ and out of $\Omega$.

Using a model reaction rate of Arrhenius type, Pismen and Kharkats [4] were the first to point out the existence of asymmetric or nonconstant steady states for the problem in the slab $\{(x, y, z) 10<x<L\}$ while assuming $r_{1}=c_{2}$ and $r_{1}=r_{2}$, and $c(x, y, z)=c(x)$, in short, a symmetric problem Aronson and Peletier [1] and Luss et al. [3] discuss the stability of such solutions. In this paper we will discuss the existence and stability of similar radically symmetric, nonconstant solutions to the problem (1) with $\Omega^{\star}$ being a cylindrical or spherical shell.

The model reaction rates $r_{j}(c)$ are usually assumed to be of Arrhenius type, but for the sake of computational simplicity when we do computations we will assume a model rate attributed to Marek [1],

$$
r_{j}(c)=\frac{k_{j}^{1 *} c}{\left(1+k_{j}^{2 \star} c\right)^{2}}, \quad k_{j}^{1 *}, k_{j}^{2 *}>0 .
$$

We will denote by $R_{1}^{\star}$ and $R_{2}^{\star}$ the inner and outer radii, respectively, of the cylindrical or spherical shell $\Omega^{\star}$. If we introduce the dimensinrless variables $t=\left(n /\left(R_{2}^{\star}-R_{1}^{\star}\right)^{2}\right) t^{\star},(x, y, z)=\left(1 /\left(R_{2}^{\star}-R_{1}^{\star}\right)\right) \cdot\left(x^{\star}, y^{\star}, z^{\star}\right), u(x, y, z, t)$ $=C\left(\left(R_{2}^{\star}-R_{1}^{\star}\right)(x, y, z),\left(\left(R_{2}^{\star}-R_{1}^{\star}\right)^{2} / D\right) \cdot t\right)$, then $u$ satisfies the differential equation

$$
\begin{aligned}
& \text { a) } u_{t}=\Delta u \text { in } \Omega, \\
& u(x, y, z, 0)=u_{0}(x, y, z),
\end{aligned}
$$

b) $\frac{\partial u}{\partial n}+\lambda f_{j}(u)=0$ on $\partial \Omega_{j}$,

with $\lambda=k\left(R_{2}^{\star}-R_{1}^{\star}\right) / D$, and

$$
f_{j}(u)=u-\frac{c_{j}}{c_{1}}+\frac{k_{j}^{1 \star} u}{\left(1+k_{j}^{2 \hbar} c_{1} j\right)^{2}}
$$

$$
=u-k_{j}^{3}+\frac{k_{j}^{1} u}{\left(1+k_{j}^{2} u\right)^{2}} \text {. }
$$


Here $K_{j}^{1}=K_{j}^{1 *}, k_{j}^{2}=C_{1} K_{j}^{2 *}$ and $K_{j}^{3}=C_{j} / C_{1}$.

Construction of Solutions

We will look for nonconstant steady state solutions of (3) for the cylindrical shell $\Omega=\left\{(x, y, z) \mid R_{1}^{2}<x^{2}+y^{2}<R_{2}^{2}\right\}$ that have the property $u(x, y, z)=v(\rho)$, where $\rho=\sqrt{x^{2}+y^{2}}$. The only functions harmonic in $\Omega$ having this property are of the form $u(x, y, z, t)=a \cdot \log (p)+b$. If we specify $u$ to be $p$ on $\partial \Omega_{1}=\left\{\rho=R_{1}\right\}$ and to be $q$ on $\partial \Omega_{2}=\left\{\rho=R_{2}\right\}$, we have

$$
u=\frac{q-p}{\log \left(R_{2} / R_{1}\right)} \log (\rho)+\frac{\log \left(R_{2}\right) p-\log \left(R_{1}\right) q}{\log \left(R_{2} / R_{1}\right)} .
$$

Substituting this value for $u$ into the boundary conditions $3(b)$, we find

$$
\begin{aligned}
& -\frac{(q-p)}{\operatorname{Tog}\left(R_{2} / R_{1}\right)} \cdot \frac{1}{R_{1}}+\lambda f_{1}(p)=0 \text { on } \partial \Omega_{1} \\
& \frac{(q-p)}{\operatorname{Tog}\left(R_{2} / R_{1}\right)} \cdot \frac{1}{R_{2}}+\lambda f_{2}(q)=0 \text { on } \partial \Omega_{2}
\end{aligned}
$$

or

$$
\begin{aligned}
& q=p+R_{1} \log \left(R_{2} / R_{1}\right) \lambda f_{1}(p) \\
& p=q+R_{2} \log \left(R_{2} / R_{1}\right) \lambda f_{2}(q) .
\end{aligned}
$$

For a steady state solution to exist we must therefore have

or

$$
0=\lambda \log \left(R_{2} / R_{1}\right)\left(R_{1} f_{1}(p)+R_{2} f_{2}(q)\right)
$$

$$
\left(R_{1} / R_{2}\right) f_{1}(p)+f_{2}(q)=0
$$

and also

or

$$
2(q-p)=\lambda \log \left(R_{2} / R_{1}\right)\left(R_{1} f_{1}(p)-R_{2} f_{2}(q)\right)
$$

$$
\lambda=\frac{2(a-p)}{R_{2} \log \left(R_{2} / R_{1}\right)\left(\left(R_{1} / R_{2}\right) f_{1}(p)-f_{2}(q)\right)} \text {. }
$$

In view of $(6),(7)$ may be rewritten as 


$$
R_{2} \log \left(R_{2} / R_{1}\right) \lambda=-\frac{(q-p)}{f_{2}(q)}
$$

Fixing $\lambda$ and finding the possible values of $p$ and $q$ can be difficult, and we will do as others have done and graph $\lambda$ against $q$.

This is done by pirkinq $q$ and finding the roots $p$ of (6). There are at most three positive roots, because our reaction function $f_{j}$, like the Arrhenius reaction, is differentiable and increases monotonically from $f_{j}(0)<0$ to a local maximum at a point we will denote by $\alpha_{j}$, then decreases monotonically to a local minimum at $\beta_{j}$ and then increases monotonically to $\infty$. Such model equation will be called "suitable," and unless stated otherwise all of the following analyses holds for any "suitable" model equation. For each root $p$ we may calculate a $\lambda$ from (7), which must be positive to have physical significance.

We will have found a constant steady state solution if $p=q$, i.e. $\left(R_{1} / R_{2}\right) f_{1}(p)+f_{2}(p)=0$. This will be a steady state only for $\lambda=0$ if $f_{1}(p) \neq 0$, or for any $\lambda \geq 0$ if $f_{1}(p)=0$. In particular this second case is true if $f_{1}=f_{2}$.

We have derived the equation for a cylindrical shell. For the spherical shell $\Omega=\left\{R_{1}^{2} \leq x^{2}+y^{2}+z^{2}=\rho^{2} \leq R_{2}^{2}\right\}$ we may derive a similar set of equations, to wit

$$
\begin{aligned}
& \left(R_{1} / R_{2}\right)^{2} f_{1}(p)+f_{2}(q)=0 \\
& \left(R_{2} / R_{1}\right) \lambda=-\frac{(q-p)}{f_{2}(q)} .
\end{aligned}
$$

Note that both sets of equations are of the form

$$
\begin{aligned}
& \left(R_{1} / R_{2}\right)^{n-1} f_{1}(p)+f_{2}(q)=0 \\
& \mu=-\frac{(q-p)}{f_{2}(q)}
\end{aligned}
$$

where $n=3$ for the spherical shell and $n=2$ for the cylindrical shell (and $n=1$ for the slab, for that matter), and so for each value of $\left(R_{c} / R_{1}\right)^{n-1}$ we 
will graph $\mu$ against $q$. The correspondence between $\lambda$ and $\mu$ is easy to see for each domain.

Numerical Results

A series of diagrams of $\mu$ versus $q$ for the value of the parameter $\left(R_{1} / R_{2}\right)^{n-1}=2 / 3$ using our specific model equations is given in figures $1-7$.

The branches of $\mu$ where the steady state solutions of tire problem for the spherical shell are stable are indicated by solid lines, the rest by dashed lines. We make the conjecture that the solutions for the cylindrical shell have the same region of stability. The stability analyses are given in the next section.

The first set of diagrams were drawn with $f_{1}=f_{2}$ (consequently $\left.k_{1}^{3}=k_{2}^{3}=1\right)$. The value of $k^{1}=k_{1}=k_{\frac{1}{2}}^{1}$ was kept fixed, and the value of $K^{\frac{1}{2}}=K_{1}^{2}=K_{2}^{2}$ was changed to indicate the qualitative behavior of the $\mu$ vs. a diagrams. The values of $k^{1}$ and $k^{2}$ were chosen so that $f_{1}$ has three real positive roots. These roots are all necessarily between 0 and 1 hecause $f_{1}(0)=-1$ and the curve is otherwise above the line $y=u-1$.

Each of these roots provides us with a steady state solution for all $\mu$, and these are indicated by the vertical lines in the diagram. As indicated, the two outside lines are stable, and the inner one is not.

In each diagram there is at least one interval, and sometimes two, where there are no steady state solutions $u$ of (3) with the value $q$ on the outside surface of the cylindrical or spherical shell for any positive $\mu$. This can happen quite generally, even if $f_{1} \neq f_{2}$. We will assume that $f_{1}$ is not necessarily equal to $f_{2}$, but that both are "suitable" and that the position of the local extremes of $f_{2}, \alpha_{2}$ and $\beta_{2}$, are contained in the smallest interval containing the roots of $f_{1}(x)=0$. (This assumption al iows us to ignore the behavior of $f_{1}$ and $f_{2}$ outside of the smallest intervals containing their respective zeros.) Then gaps occur if

$$
\text { (a) }\left(R_{1} / R_{2}\right)^{n-1} f_{1}\left(\alpha_{1}\right)+f_{2}\left(\beta_{2}\right)<0 \text {, or }
$$

(b) $\left(R_{1} / R_{2}\right)^{n-1} f_{1}\left(B_{1}\right)+f_{2}\left(a_{2}\right)>0$. 
If (a) is true then there is an interval around $\beta_{2}$ for which there corresponds no positive $\mu$ for any $q$ in the interval, because the solution of (9) yielding positive $\mu$ is impossible for such $q$; similarly if (b) is true. If there is equality in (a) then there is a critical point at $\beta_{2}$, and similarly for (b).

If we consider our specific model equation for the reaction rate and assume that $f_{1}=f_{2}$, then there is a particularly elegant description of when equality in (15)a or (15)b will occur. As was pointed out by Kaper, et al. [2], for each value of $k^{1}$ there is a range of $k^{2}$, depending on $k^{1}$, such that if $k_{L}^{2}<k^{2}<k_{u}^{2}$ then $f$ has exactly three positive real roots. This set $\left\{\left(k^{1}, k^{2}\right) \mid f\right.$ has three positive real roots $\}$ forms a wedge, the vertex of which is $(27,8)$. (Recall $\left.k_{1}^{3}=k_{2}^{3}=1.\right)$ The upper and lower boundaries are asymptotically

$$
\begin{aligned}
& K_{u}^{2}=\frac{1}{4} k^{1}+1+0\left(1 / K^{1}\right) \quad \text { as } K^{1}+\infty \text { and } \\
& K_{L}^{2}=2\left(K^{1}\right)^{1 / 2}-2+0\left(\left(k^{1}\right)^{1 / 2}\right) \text { as } K^{1}+\infty .
\end{aligned}
$$

For $k^{2}=k_{L}^{2}, f_{1}$ has a double root at $\beta_{1}$, and for $k^{2}=k_{u}^{2}, f_{1}$ has a double root at $\alpha_{1}$. Kaper et al. found that, for the slab, their single condition for a critical point was

$$
f_{1}\left(\alpha_{1}\right)+f_{1}\left(\beta_{1}\right)=0
$$

and that this occurred when $k^{2}$ was exactly equal to the average of $K_{L}^{2}$ and $K_{u}^{2}$. In our case the situation is completely analogous. We state, without proof, the following result.

Proposition: a) There is a critical point at $q=\alpha_{1}$ if

$$
k^{2}=\frac{k_{L}^{2}+\left(R_{1} / R_{2}\right)^{n-l_{1} k_{u}}}{1+\left(R_{1} / R_{2}\right)^{n-1}} .
$$

b) There is a critical point at $q=\beta_{1}$ if

$$
k^{2}=\frac{\left(R_{1} / R_{2}\right)^{n-1} k_{L}^{2}+k_{u}^{2}}{\left(R_{1} / R_{2}\right)^{n-1}+1} .
$$


This problem (3) for the cylindrical or spherical shell is singular in the following sense. If $f_{2}$ is any perturbation of $f_{1}$ such that $f_{1}$ and $f_{2}$ have different roots, then there is a salution of

$$
\left(R_{1} / R_{2}\right)^{n-1} f_{1}\left(q_{0}\right)+f_{2}\left(q_{0}\right)=0
$$

for which $\mathrm{f}_{2}\left(\mathrm{q}_{0}\right) \neq 0$, and so $\mu=0$ for this (constant) steady state. Because of at least one sided continuity at the point $\left(q_{0}, 0\right)$, there are arbitrarily small positive $\mu$, and hence $\lambda$, for which there exist nonconstant steady state solutions of (3). This is false for the case $f_{1}=$ $f_{2}$, for which there is a minimum $\mu_{0}>0$ such that, if $\mu<\mu_{0}$, there is no nonconstant steady state solutions corresponding to $\mu$. This phenomenon is illustrated in [2].

Because the $\mu$ versus $p$ curves are qualitatively different from the $\mu$ versus $q$ curves, we include also several $\mu$ vs. $p$ diagrams. Their qualitative behavior can be explained in a manner similar to the $\mu$ vs. q diagrams.

Stability

In this section we assume only that the $f_{j}$ 's are differentiable. A linear stability analysis about a steady state solution $u$ of (3) requires the study of the stability of the linearized equation

$$
\begin{aligned}
& v_{t}=\Delta v \text { in } \Omega, \\
& v(x, y, z, 0)=v_{0}(x, y, z), \\
& \frac{\partial v}{\partial n}+\lambda f_{j}^{\prime}(u) v=0 \text { on } \partial \Omega_{j} .
\end{aligned}
$$

From variational principles we know that all solutions $v$ of (18) will converqe to 0 if $f_{j}^{\prime}(u)>0$ everywhere, and some will blow up if $f_{j}(u)<0$ everywhere. Thus, the solution $u$ of $(3)$ is stable if $f_{j}^{\prime}(u)>0$ everywhere, and is unstable if $f_{j}^{\prime}(u)<0$ everywhere. We would like to find the other regions of stability for our special domains $\Omega$ and steady states $u$.

For the steady state solutions $u(x, y, z, t)=p+(q-p) x$ for problem (3) in the slab $\Omega=\{(x, y, z) \mid 0<x<1\}$ with $\partial \Omega_{1}=\{x=0\}, \partial \Omega_{2}=\{x=1\}$, we find strability if and only if 


$$
e^{2 \sigma}=\frac{\left(\lambda f_{2}^{\prime}(q)-\sigma\right)\left(\lambda f_{1}^{\prime}(p)-\sigma\right)}{\left(\lambda f_{2}^{\prime}(q)+\sigma\right)\left(\lambda f_{1}^{\prime}(p)+\sigma\right)}
$$

has no nonzero real solution $\sigma$. This condition is fulfilled if and only if

$$
\begin{aligned}
& \text { a) } f_{2}^{\prime}(q)>0 \text { and } f_{i}(p)>0 \text {, or } \\
& \text { b) } \lambda+\frac{1}{f_{1}^{\prime}(p)}+\frac{1}{f_{2}^{\prime}(q)}<0 \text { if } f_{1}^{\prime}(p) f_{2}^{\prime}(q)<0 \text {. }
\end{aligned}
$$

(See Pismen and Kharkats [4].)

We may derive a similar expression for the problem (3) in the spherical shell.

We assume a solution of the form $v(x, y, z, t)=e^{\sigma^{2} t} w(x, y, z)$, where $\sigma^{2}$ is real, positive or negative, because $(18)$ is a selfadjoint problem. We may derive an equation for $w$

$$
\begin{array}{ll}
\text { a) }-\Delta w+\sigma^{2} w=0 & \text { in } \Omega \\
\text { b) } \frac{\partial w}{\partial r}+\lambda f_{j}^{\prime}(u) w=0 & \text { on } \partial \Omega_{j} .
\end{array}
$$

Now $w$ must be radially symmetric, i.e. $w=w(\rho)$, since $\Omega$, the differential operator, and the boundary conditions are radially symmetric and the problem is linear.

The solution w therefore is of the form

$$
w(\rho)=a(\sigma \rho)^{-1} e^{\sigma \rho}+b(\sigma \rho)^{-1} e^{-\sigma \rho} .
$$

Substituting this into the boundary conditions $20(\mathrm{~b})$ and denoting $\lambda f_{1}(p)$ by $q_{1}$, and $\lambda f_{2}^{\prime}(q)$ by $g_{2}$ we require

$$
\begin{aligned}
& \frac{a}{R_{2}} e^{\sigma R_{2}}\left(1+\frac{R_{2} g_{2}-1}{\sigma R_{2}}\right)+\frac{b}{R_{2}} e^{-\sigma R_{2}}\left(-1+\frac{R_{2} g_{2}-1}{\sigma R_{2}}\right)=0 \\
& \frac{a}{R_{1}} e^{\sigma R_{1}}\left(-1+\frac{R_{1} g_{1}+1}{\sigma R_{1}}\right)+\frac{b}{R_{1}} e^{-\sigma R_{1}}\left(1+\frac{R_{1} g_{1}+1}{\sigma R_{1}}\right)=0 .
\end{aligned}
$$


If this set of linear equations has a nonzero solution $(a, b)$ for some nonzero real $\sigma$, then $\sigma^{2}$ will be positive and $v$ will be unbounded in time. Thus we wish to see, for fixed values of $R_{1}$ and $R_{2}$, for which values of $g_{1}$ and $a_{2}$ is there a real nonzero o such that the determinant of (21) is zero.

This condition can be written as

$$
e^{2 \sigma}=\frac{\left(\frac{R_{2} g_{2}-1}{R_{2}}-\sigma\right)\left(\frac{R_{1} g_{1}+1}{R_{1}}-\sigma\right)}{\left(\frac{R_{2} g_{2}-1}{R_{2}}+\sigma\right)\left(\frac{R_{1} g_{1}+1}{R_{1}}+\sigma\right)} .
$$

(Recall $\left.R_{2}-R_{1}=1.\right)$

For stability we require that there be no real roots $\sigma$ of this equation. This occurs if and only if

a) $R_{2} g_{2}-1>0$ and $R_{1} g_{1}+1>0$, or

b) $\left(R_{2} g_{2}-1\right)\left(R_{1} g_{1}+1\right)<0$ and

$$
\text { 1. } \leq-\left(\frac{R_{2}}{R_{2} a_{2}-1}+\frac{R_{1}}{R_{1} a^{1}+1}\right) \text {. }
$$

(Compare with (19).)

These conditions can be reformulated as

a) $q_{2}=\lambda f_{2}^{\prime}(q)>0$ and $g_{1}=\lambda f_{1}^{\prime}(p)>0$, or

b) $f_{2}^{\prime}(q) f_{1}^{\prime}(p)<0$ and

$$
\frac{R_{2}}{R_{1}} \lambda+\frac{1}{\left(R_{1} / R_{2}\right)^{2} f_{1}^{\prime}(p)}+\frac{1}{f_{2}^{\prime}(q)} \leq 0 .
$$

It was pointed out by Aronson and Peletier [1] for $f_{1}=f_{2}$ for the slab that condition (19)b is equivalent to the statement that $f_{1}^{\prime}(p) f_{2}^{\prime}(q)$ $\left\langle 0\right.$ and $\frac{d \lambda}{d q}>0$. It is an easy derivation from $\left(6^{\prime}\right)$ and $\left(8^{\prime}\right)$ to conclude the following result. 
Proposition. The radially symmetric steady state solution u of (3), where $\Omega$ is a spherical shell, taking the values $p$ and $q$ on the inner and outer surfaces of radii $R_{1}$ and $R_{2}$ respectively is stable to small perturbations if and only if

$$
\text { a) } f_{1}^{\prime}(p)>0 \text { and } f_{2}^{\prime}(q)>0 \text { or }
$$

b) $f_{1}^{\prime}(p) f_{2}^{\prime}(q)<0$ and $\frac{d \lambda}{d q}>0 \quad / / /$

A simiiar analysis may be made when $\Omega$ is a cylindrical sheil. There the analysis is complicated because the form of $w$ is

$$
w=\mathrm{aI}_{0}(\sigma \rho)+\mathrm{bK}_{0}(\sigma \rho)
$$

where $I_{v}(z)$ and $K_{v}(z)$ are modified Bessel functions of order $v$. The analysis gives

Proposition. The radially symmetric steady state solution $u$ of (3), with $\Omega$ a cylindrical shell, taking the yaiues $p$ and $q$ on the inner and outer surfaces of radii $R_{1}$ and $R_{2}$ respectively is stable to small perturbations if and only if:

there is no real solution of the equation

$$
\begin{aligned}
& {\left[\lambda f_{1}^{\prime}(p) K_{0}\left(R_{1} \sigma\right)+\sigma K_{1}\left(R_{1} \sigma\right)\right] \times\left[\lambda f_{2}^{\prime}(q) I_{0}\left(R_{2} \sigma\right)+\sigma I_{1}\left(R_{2} \sigma\right)\right]} \\
& -\left[\lambda f_{1}^{\prime}(p) I_{0}\left(R_{1} \sigma\right)-\sigma I_{1}\left(R_{1} \sigma\right)\right] \times\left[\lambda f_{2}^{\prime}(q) K_{0}\left(R_{2} \sigma\right)+\sigma K_{1}\left(R_{2} \sigma\right)\right]=0 \quad / / /
\end{aligned}
$$

We conjecture that condition (28) is equivalent to condition (26).

\section{Limiting Cases}

As $\left(R_{1} / R_{2}\right)+1$, each of our shells looks more and more like a slab. We would therefore expect that, qiven $q$, our steady state solutions $u$, our $\lambda$ 's and the regions of stability, for each of these shells, converge to their counterpart in the slab. For each $q$ the corresponding $p, u$ and $\lambda$ for the slab will be denoted by $p_{s}, u_{s}$ and $\lambda_{s}$. 
We will have occasion to use the functions

$$
\begin{array}{ll}
h_{1}:\left[0, \alpha_{1}\right]+R & h_{1}(x)=f_{1}(x) \\
h_{2}:\left[\alpha_{1}, \beta_{1}\right]+R & h_{2}(x)=f_{1}(x) \\
h_{3}:\left[\beta_{1}, \infty\right]+R & h_{3}(x)=f_{1}(x)
\end{array}
$$

These are restrictions of $f_{1}$ that are $1-1$ and hence have well defined converses.

We will deal first with the spherical shell. We may rewrite (6') as

$$
p(q)=h_{j}^{-1}\left(f_{2}(q) \times\left(R_{2} / R_{1}\right)^{2}\right)
$$

for each branch $h_{j}$ of $f$. As $R_{1} / R_{2} \rightarrow 1$, since $h_{j}^{-1}$ is continuous $p(q)+$ $p_{s}$. We also have that

$$
\lambda=-\left(R_{1} / R_{2}\right) \frac{(q-p)}{f_{2}(q)}+-\frac{\left(q-p_{s}\right)}{f_{2}(q)}=\lambda_{s}
$$

as $R_{1} / R_{2}+1$.

What we'd like to show is that, with $u=u(\rho),\|u "\| u^{\infty}\left[R_{1}, R_{2}\right]+0$, because then $u$ would converge to the straight line joining $q$ and $p_{s}$, i.e. $u_{s}$. The solution is

$$
u(\rho)=\frac{-R_{1} R_{2}(q-p)}{\rho}+R_{2} q-R_{1} p .
$$

If we differentiate twice with respect to $\rho$ we get

$$
u^{\prime \prime}(\rho)=\frac{2 R_{1} R_{2}(p-q)}{\rho^{3}}+0 \text { for } \rho \in\left[R_{1}, R_{2}\right] \text { as } R_{1}+\infty \text {. }
$$

Hence $u+u_{s}$ in the suitable sense.

The sign of $f_{1}(p)$ is obviously the same as the sign of $f_{1}\left(p_{s}\right)$ (the opposite of the sign of $\left.f_{2}(q)\right)$, and so the question of stability is settled if $\frac{d \lambda}{d q}+\frac{d \lambda_{s}}{d q}$ as $R_{1} / R_{2}+1$. This can be shown by direct calculation.

For the case of the cylindrical shell we may show that $u \rightarrow u_{s}, p \rightarrow p_{s}$, and $\lambda+\lambda_{s}$ in a completely analogous fashion, using the form of the solution (5). Because of the form of the stability condition (28) we were not 
able to show the corivergence of the curves of stable solutions (although if our conjecture there were true, it would follow immediately, as before).

\section{Acknowledgement}

I would like to thank Hans Kaper for introducing me to this topic, and for offering me encouragement and direction.

\section{References}

1. Aronson, D. G. and Peletier, L. P., Global stability of symmetric and asymmetric concentration profiles in catalyst particles, Arch. Rat. Mech. Anal. 54, 175-204, 1974.

2. Kaper, Hans G., Leaf, Gary K., and Matkowsky, Bernard J., Asymmetric steady states in catalyst slabs, Chem. Engna Sci. 35, 2195-2201, 1980.

3. Luss, D., Bailey, J. E., and Sharma, S., Asymmetric steady states in catalytic slabs with uniform and nonuniform environments, Chem. Engng Sci. 27, 1555-1567, 1972.

4. Pismen, L. M. and Karkats, Y. I., Asymmetric statee of a heterogeneous exothermic reaction, Dokl. Akad. Nauk. SSSR 178, 901-904, 1968. 


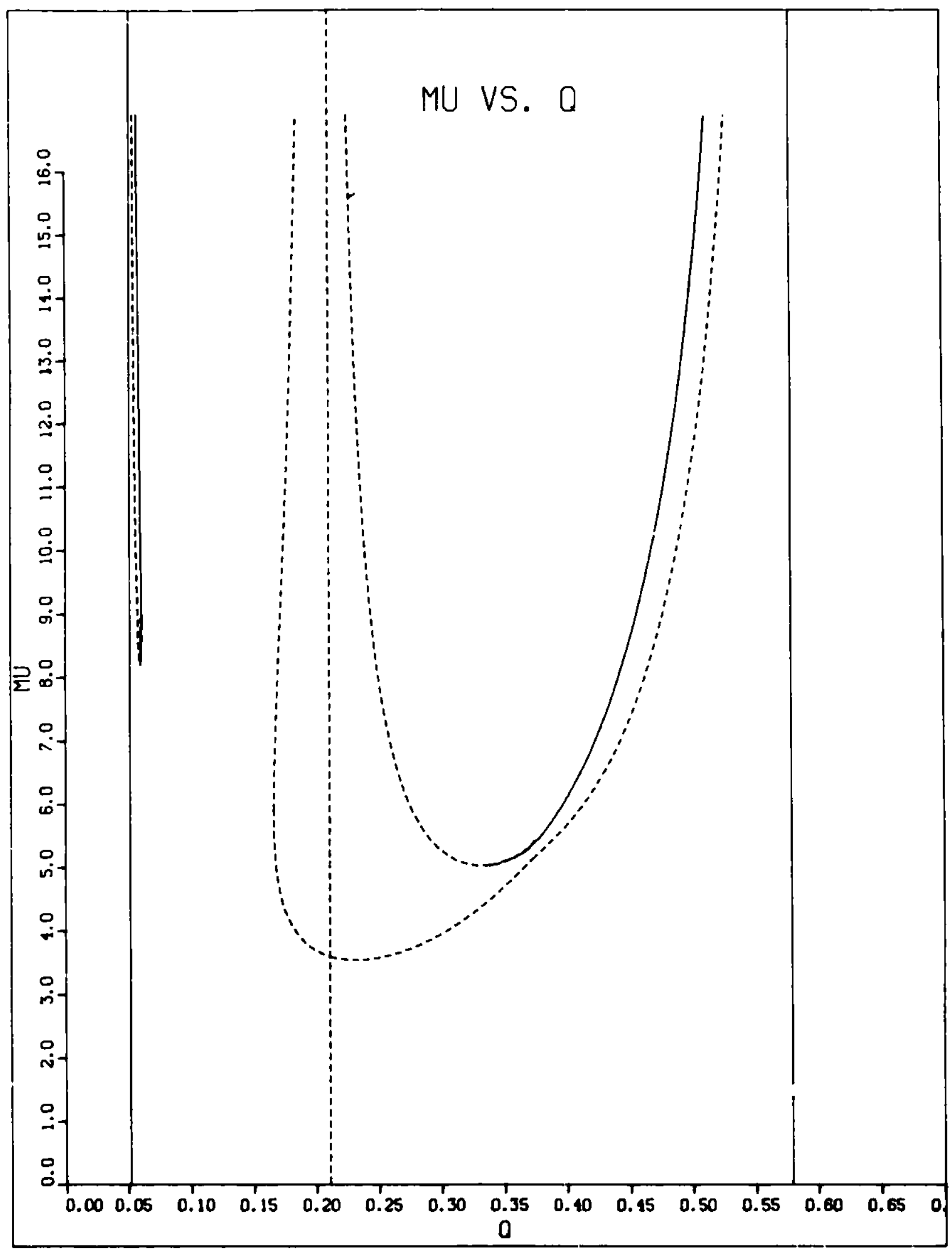

Figure 1

Solutions of $(9),(10)$ with $K_{2}=50, K_{1}=12.594684$ 
18

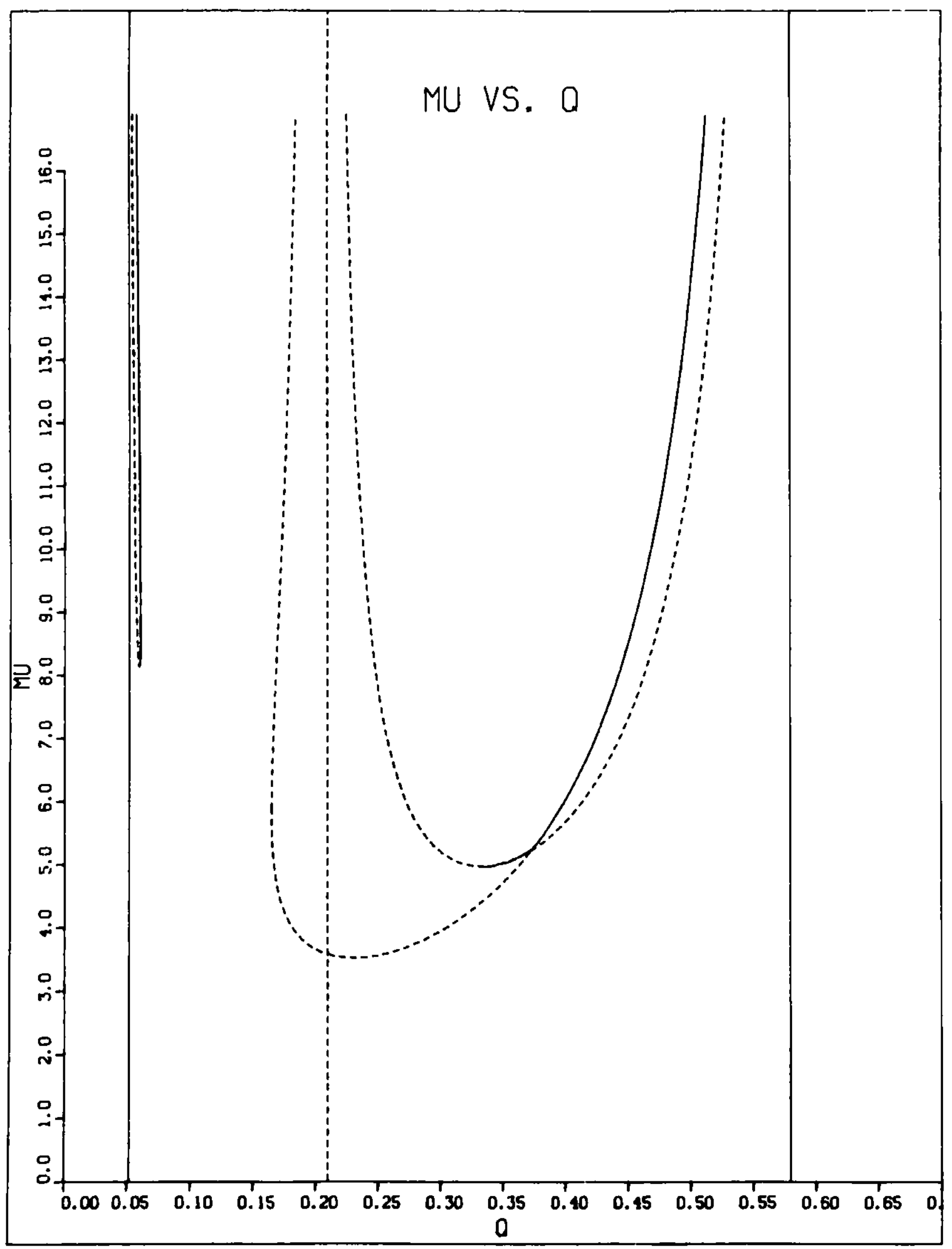

Figure 2

Solutions of $(9),(10)$ with $K_{2}=50, K_{4}: 12.598694$ 


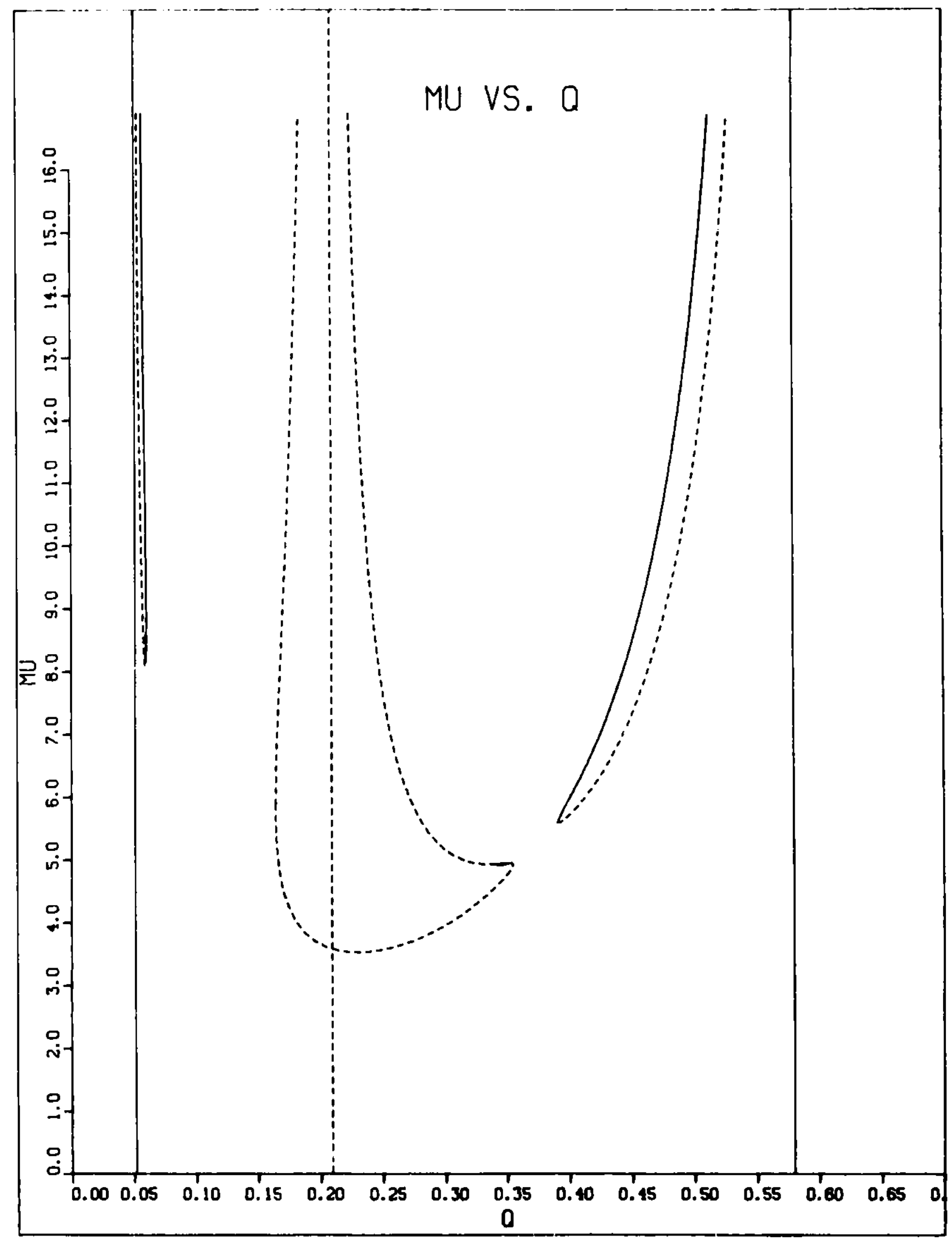

Figure 3

Solutions of $(9),(10)$ with $K_{2}=50, K_{1}=12.602673$ 


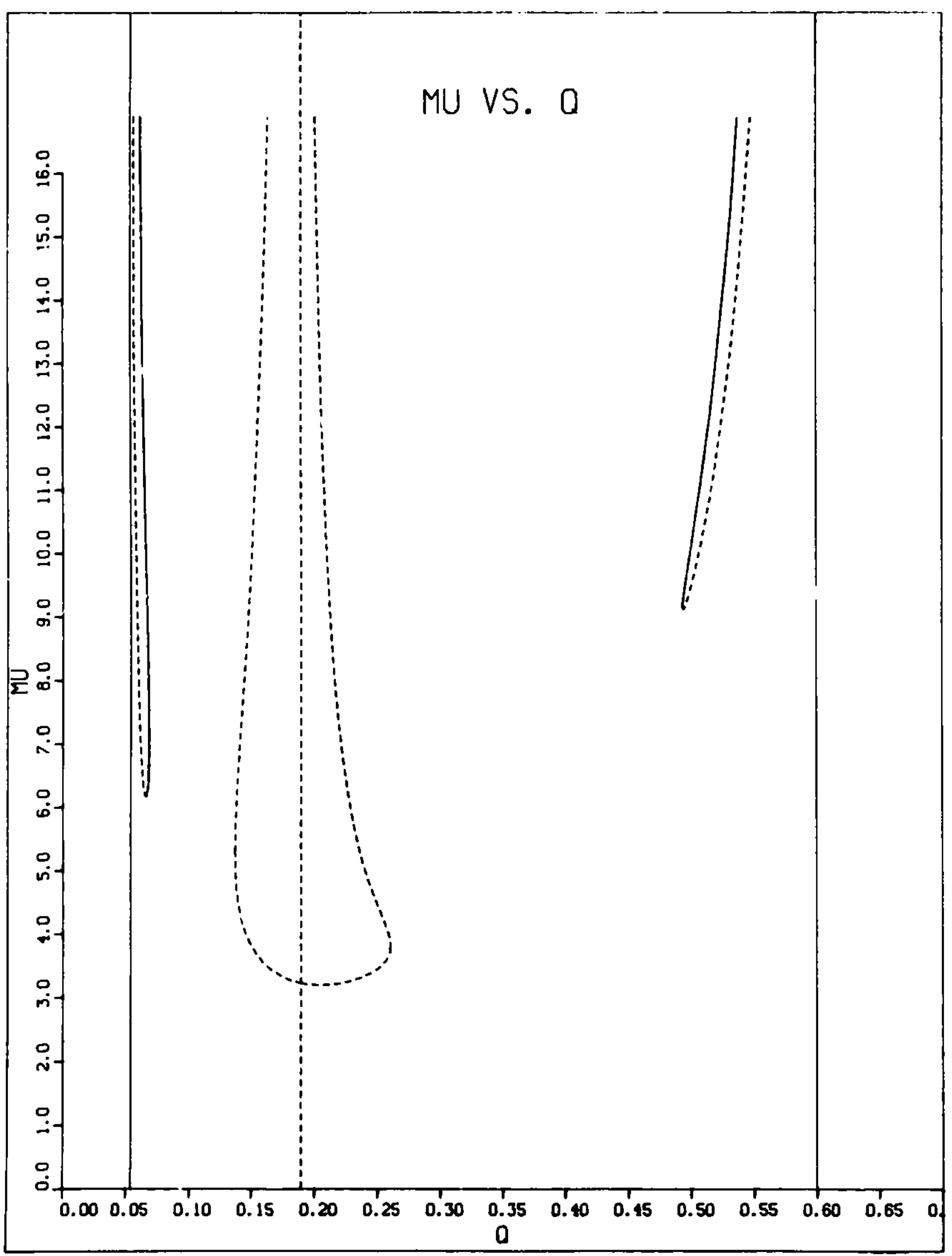

Figure 4

Solutions of (9), (10) with $K_{2}=50, K_{1}=12.765120$ 


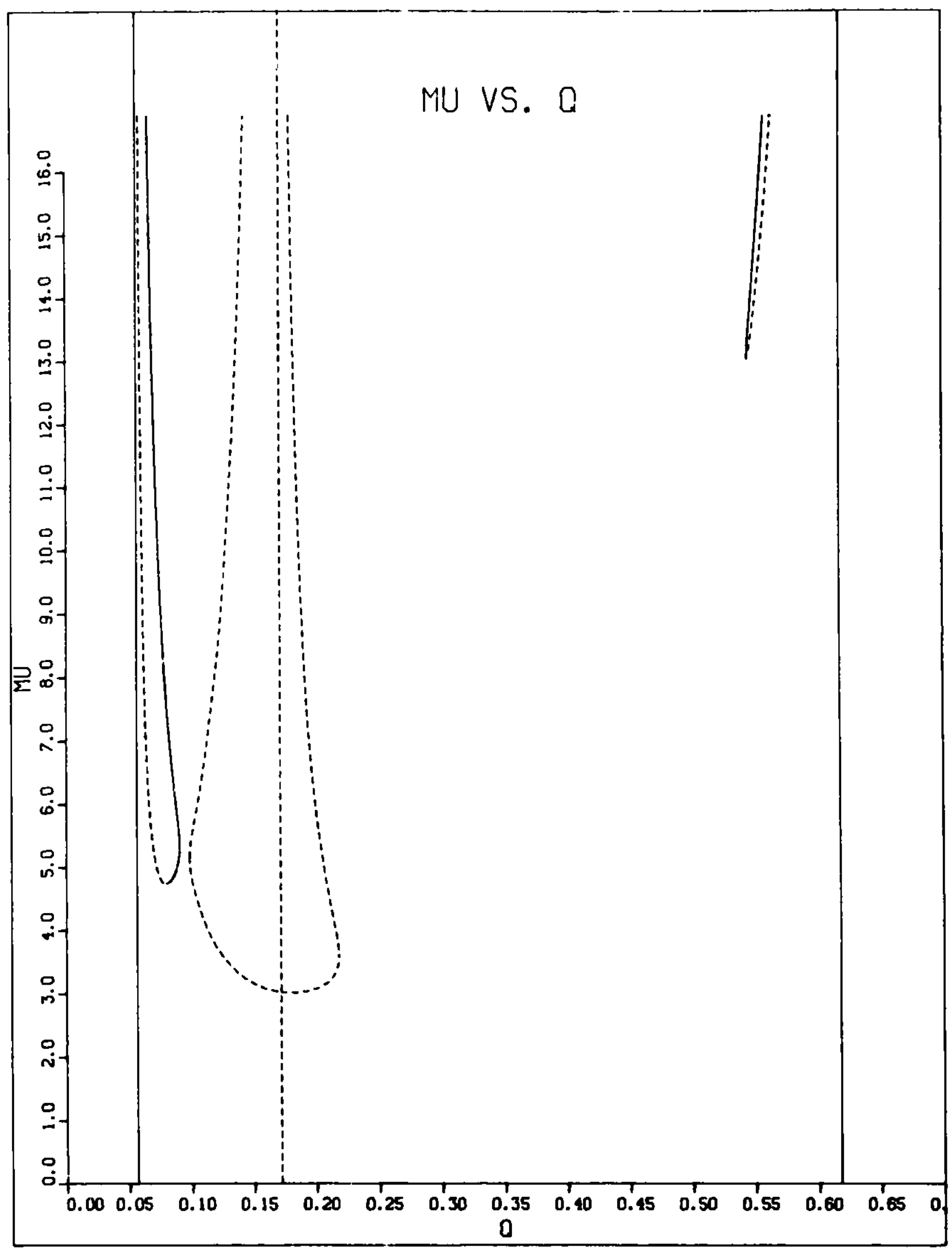

Fiqure 5

Solutions of $(9),(10)$ with $K_{2}=50, K_{1}=12.928872$ 


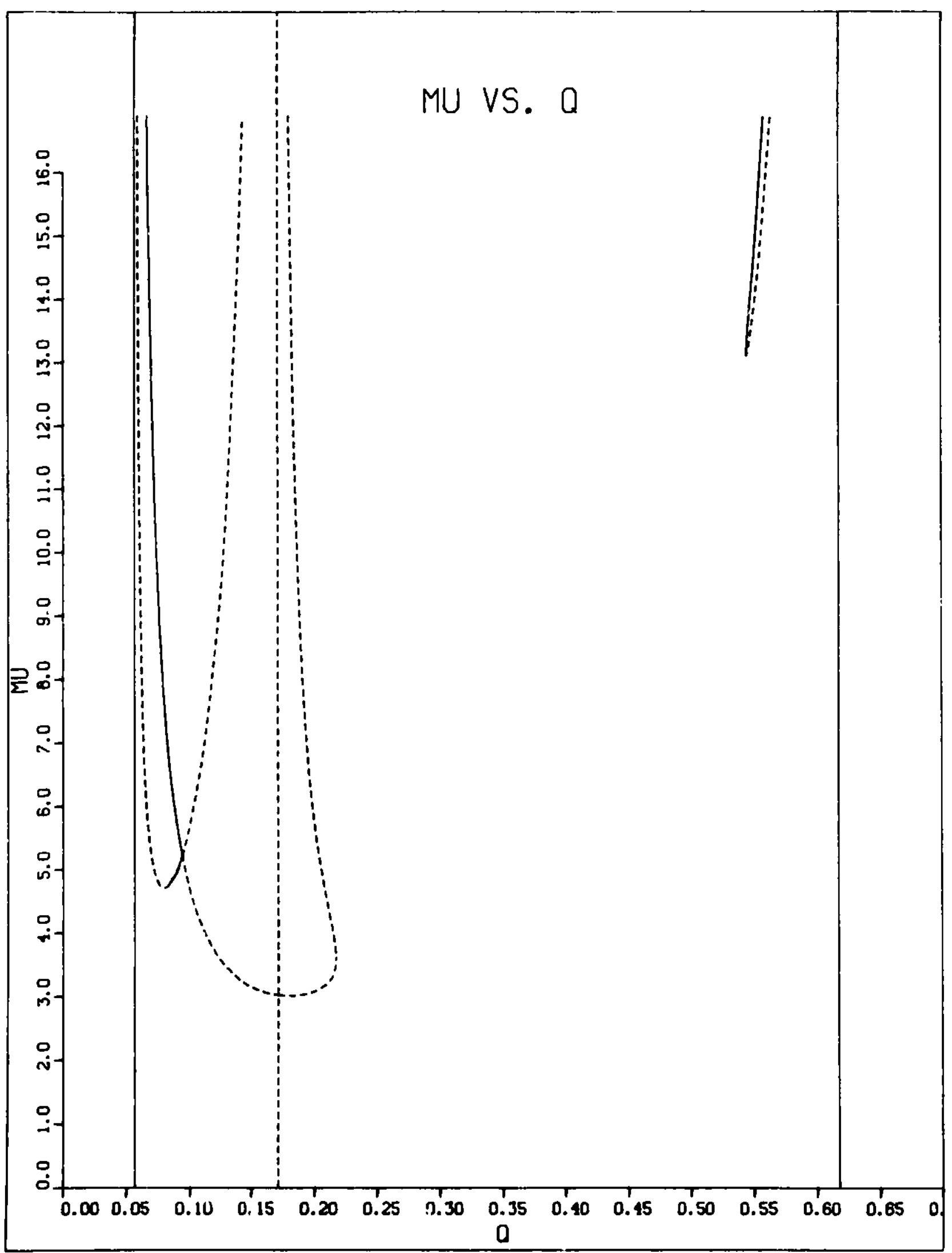

Figure 6

Solutions of $(9),(10)$ with $K_{2}=50, K_{1}=12.931540$ 


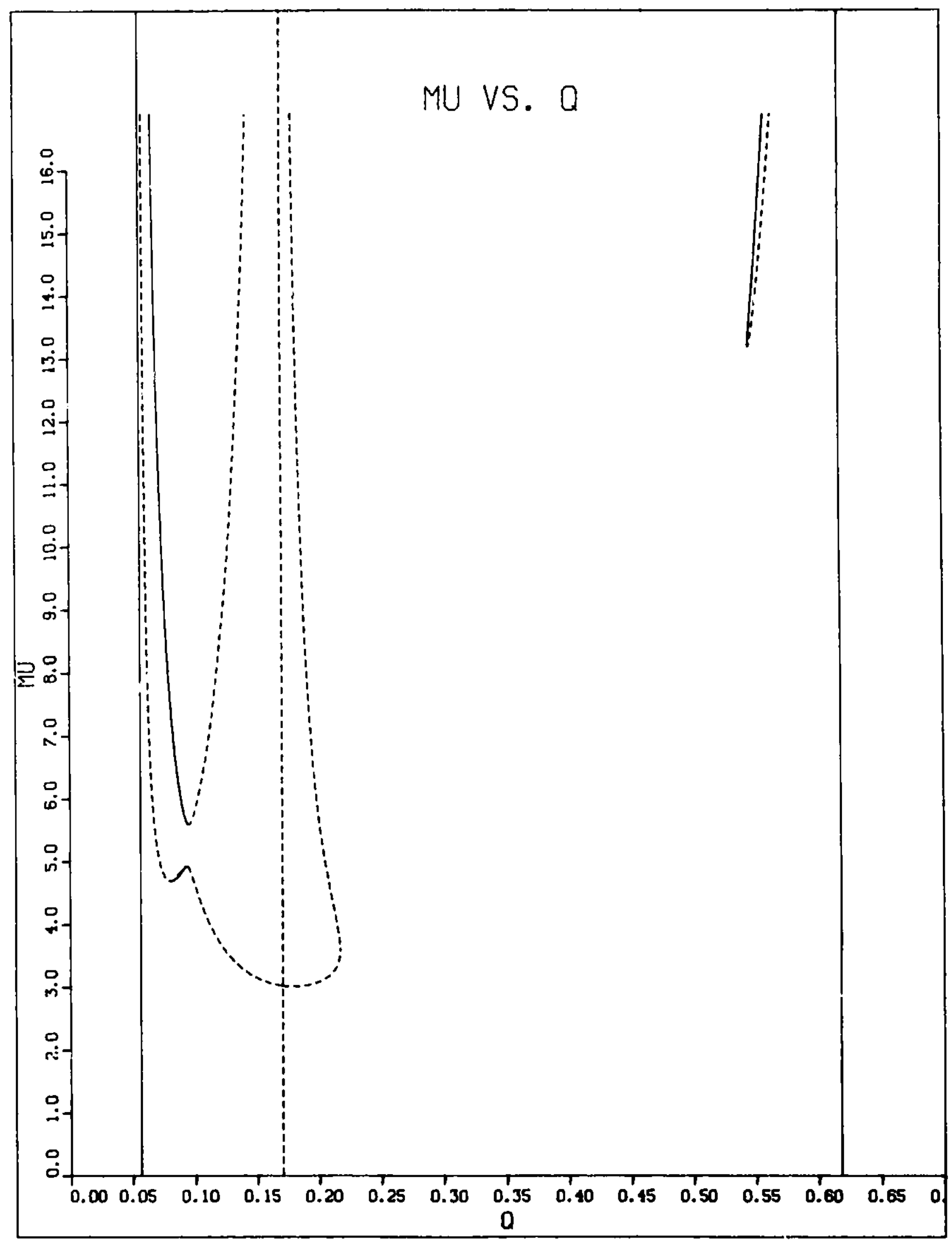

Figure 7

Solutions of $(9),(10)$ with $K_{2}=50, K_{1}=12.934137$ 


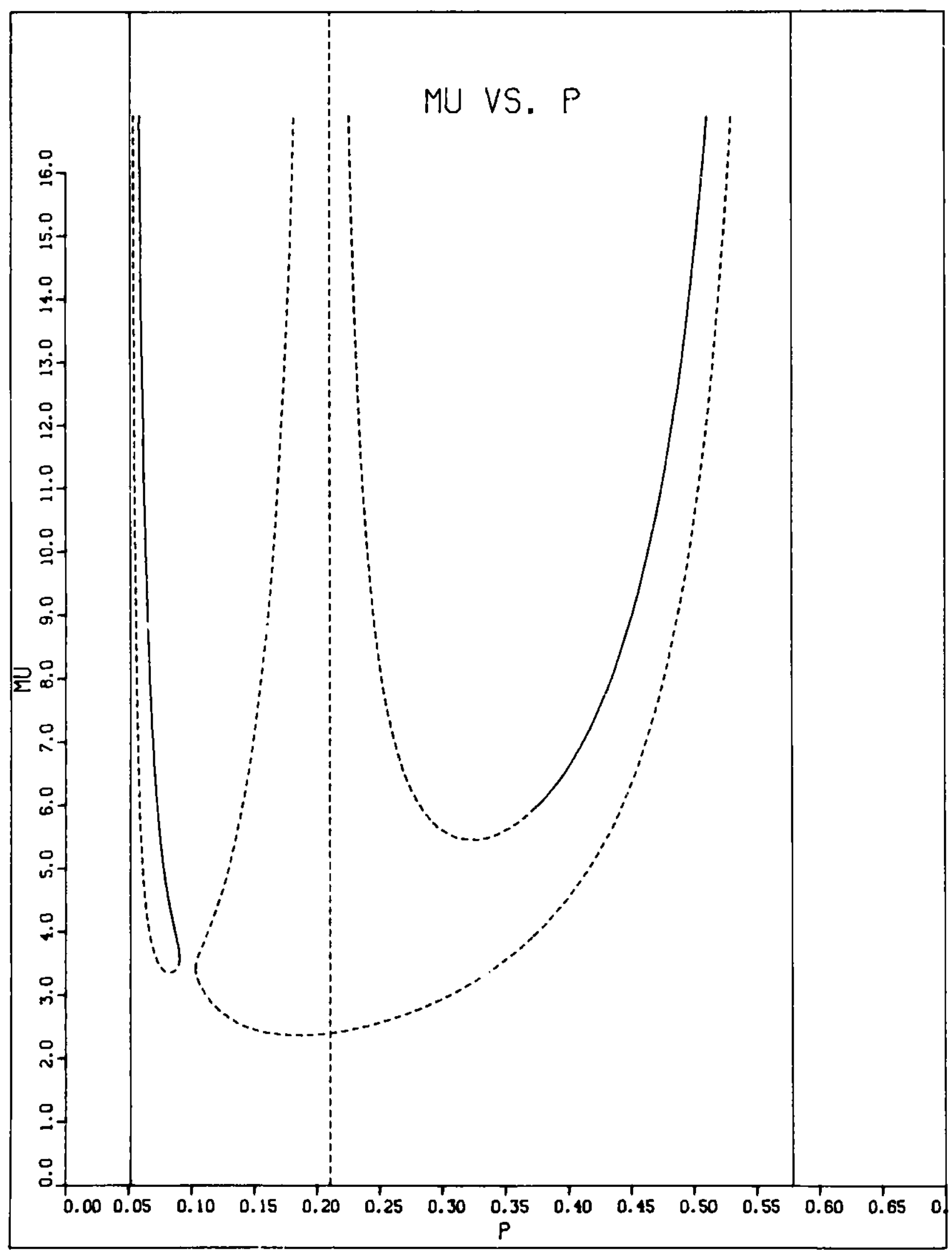

Figure 11

Solutions of (9), (10) with $K_{2}=50, K_{1}=12.594684$ 


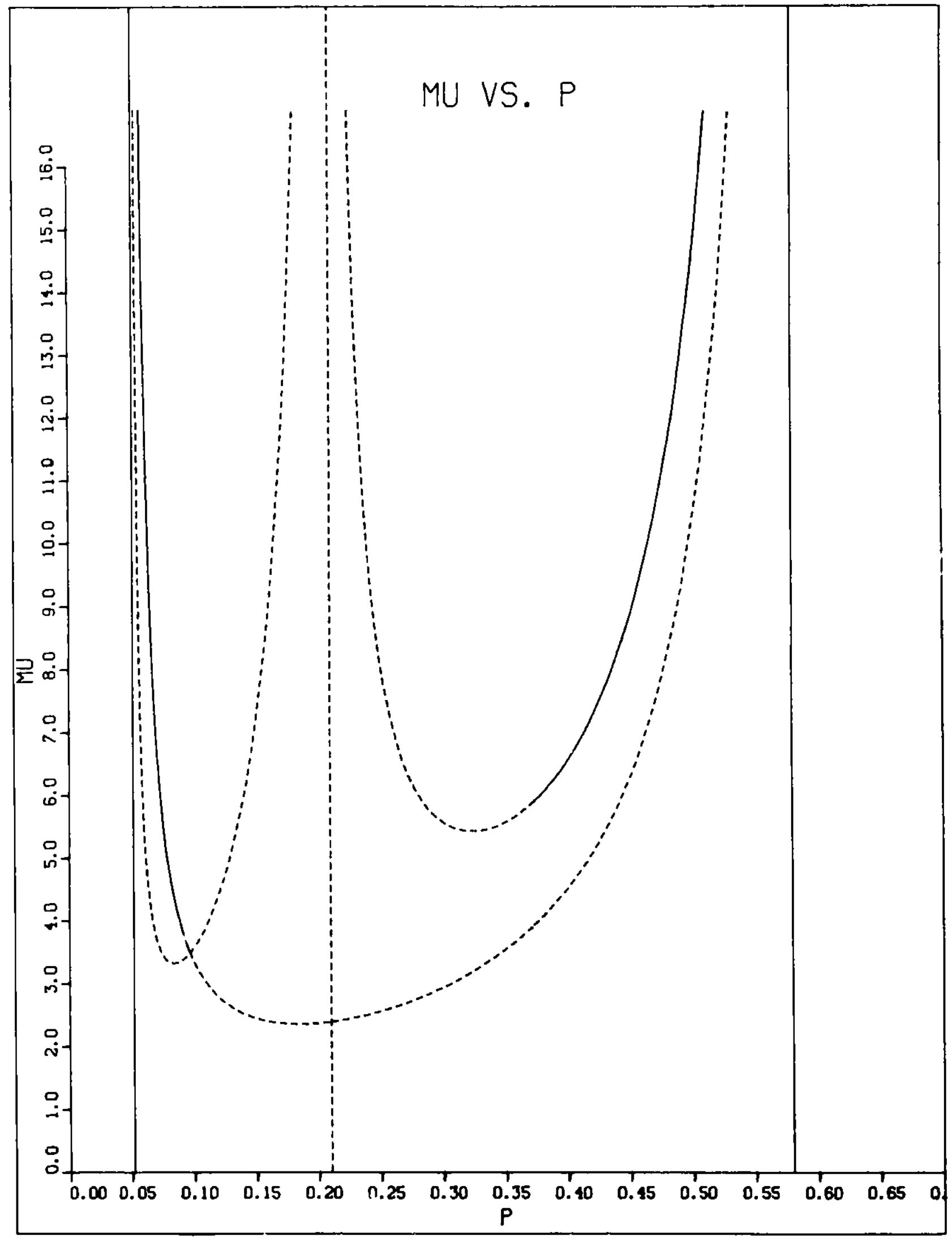

Figure 2'

Solutions of $(9),(10)$ with $K_{2}=50, K_{1}=12.598694$ 


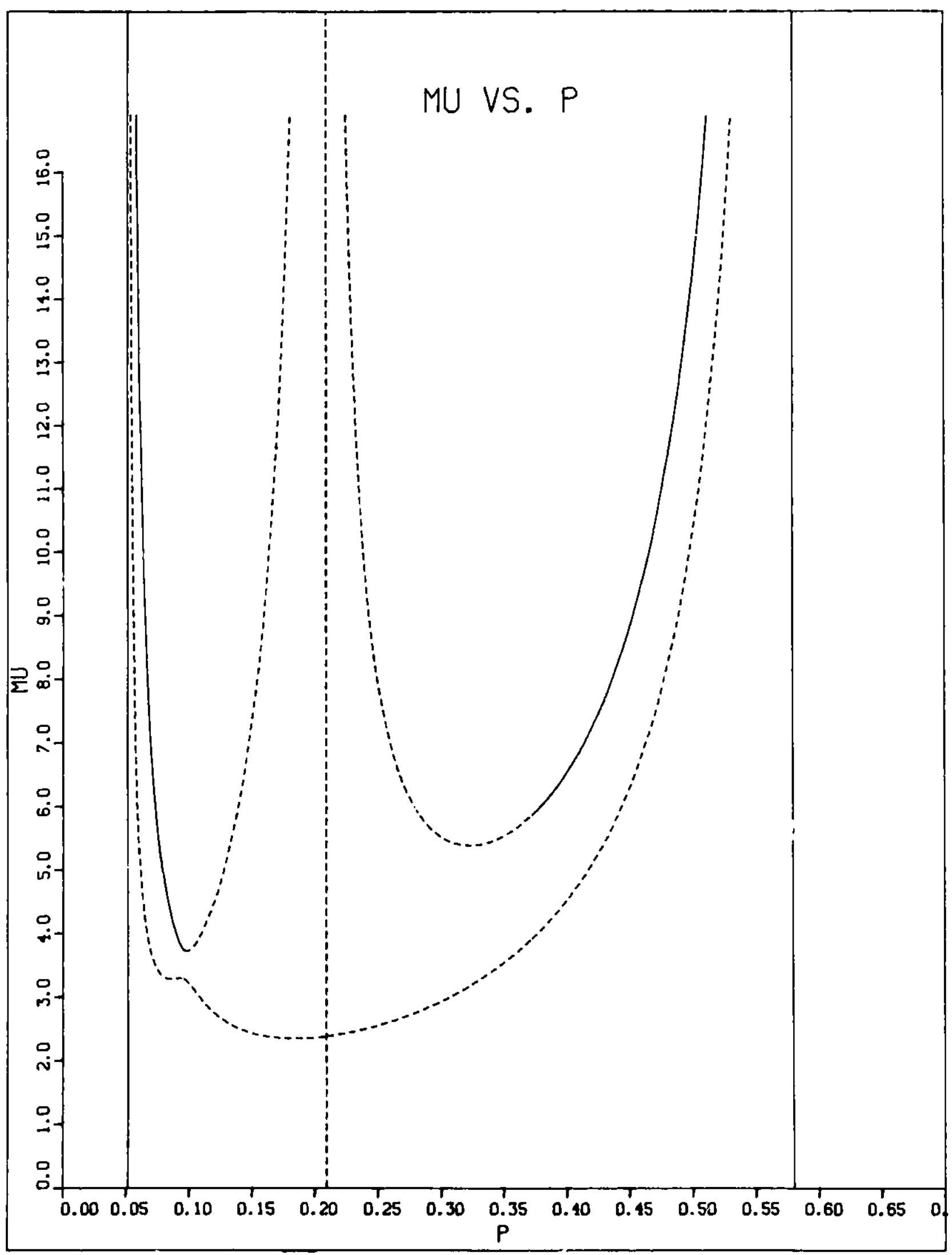

Figure $3^{\prime}$

Solutions of (9),(10) with $K_{2}=50, K_{1}=12.602673$ 


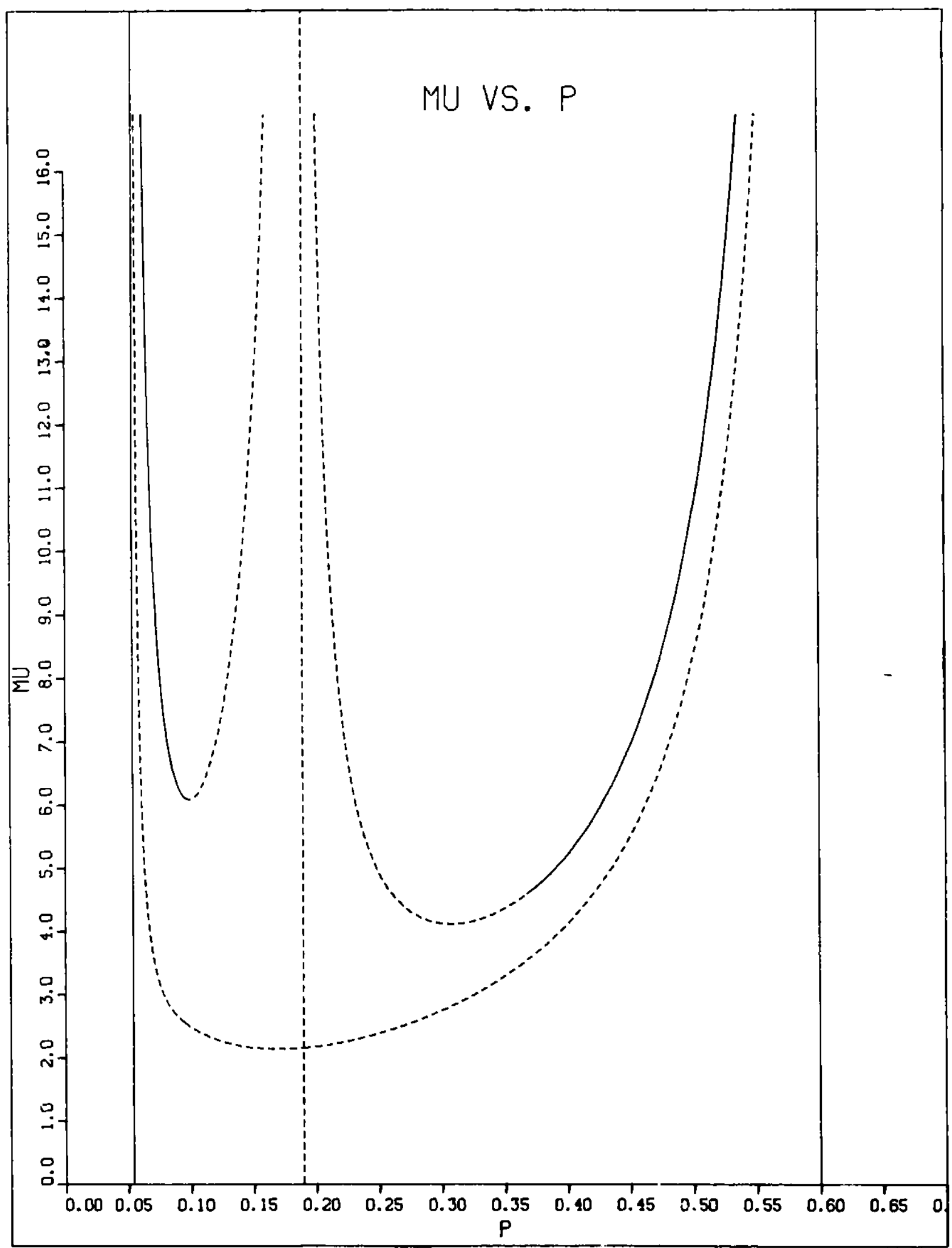

Figure $4^{\prime}$

Solutions of $(9),(10)$ with $K_{2}=50, K_{1}=12.765120$ 